\title{
Handling Conflicts in Groupware: Concepts and Experiences made in the POLITeam-Project
}

\author{
Volker Wulf \\ Institute for Computer Science III \\ University of Bonn \\ Römerstr. 164 \\ D-53117 Bonn, Germany \\ Email: volker@informatik.uni-bonn.de
}

\begin{abstract}
The activation of certain functions in groupware affects different users who might have conflicting interests. We will develop technical mechanisms to support users in regulating these conflicts. Contrary to current implementations of groupware, these mechanisms strengthen the position of the users which are affected by the activation of a function. Therefore, they provide visibility of activation, a channel for communication, or the right to intervene. After presenting the general concept, it will be shown how these mechanisms are used within the POLITeam project to support users in handling conflicts when activating certain functions.
\end{abstract}

KEYWORDS Groupware, Conflict Regulation, Evaluation, Field Study, Implementation Architecture

\section{INTRODUCTION}

In the CSCW literature it is widely assumed that cooperative work implies the existence of conflicting interests among individuals (cf. Schmidt 1991; Easterbrook et al. 1993). Conflicts may be related to a wide spectrum of different occasions and topics. In this paper a specific type of conflict will be examined. These conflicts occur when one user activates a function which produces a state transition of the groupware system. As groupware provides channels to transmit or to share data, a state transition resulting from the activation of a function might affect the interests of other users. For instance, a shared window system provides to the users a function which allows to choose among different floor control policies (cf. Greenberg 1991, pp. 21). The activation of this function to change the actual policy by one user will affect the interests of the other users of the section.
To examine conflicts resulting from the activation of groupware functions, we will distinguish among different conflict-constellations by using the concept of roles. A role describes a specific position held by users within a technically mediated interaction. In the following section we will just look at a single conflictconstellation per function: the one between the activator who uses a function actively and the role of the user affected who might suffer from disturbances caused by this action.

Conflicts resulting from the activation of a function have not yet been investigated systematically within the CSCW literature. There are only few case studies reporting empirically on this type of conflicts (cf. Cool et al. 1992; Dourish 1993; Kahler 1994). Nevertheless, looking at the design of certain applications, one can find occasionally functions equipped with a technical mechanism to regulate such conflicts among the users

Human-Computer Interaction: INTERACT'97 S. Howard. J. Hammond \& G. Lindgaard (editors)

Published by Chapman \& Hall CIFIP 1997 
(cf. chap. 2). But these mechanisms appear to be rather the result of accidental than of well grounded design decisions being aware of different alternatives.

Therefore, at first we will develop a classification scheme for technical mechanisms to support users in regulating conflicts in groupware. Furthermore, we will show how these mechanisms can be used to satisfy user requirements within a participative software development project.

\section{TECHNICAL MECHANISMS TO REGULATE CONFLICTS}

Looking at the state of the art in the CSCW literature there are two modes for handling conflicts caused by the activation of a function. They are either masked out from the ordinary users or they are solved in favour of the activator. In the first case conflicting activations of a function are solved either by a rigidly implemented automatism (e.g. concurrency control in traditional information systems) or they are solved automatically according to a specification provided by a privileged user (e.g. access control in traditional information systems) (cf. Wulf 1997). In the second case conflicting activations of a function are executed by the system in the way the activator intended it. Affected users do not get technical support to perceive the conflict or to influence the activation of a function. Whatever functional alternative the activator selects, it will be automatically executed. Thus, the system performs directly the state transition intended by the activator.

To overcome the masking out of these conflicts, their regulation has to become a design issue of the interface of ordinary users. To strengthen the role of the users affected when handling these conflicts, the deterministic relationship between the activator's choice and its execution has to be broken up.

To motivate our design approach to support users in handling this type of conflicts, we look at proposals developed in conflict theory. The underlying assumption of the mainstream in conflict theory is that the interests of the different parties involved in a conflict can be satisfied by a solution which all parties experience as mutually beneficial. Thus, it is assumed that endemic conflicts should become overt. Overt conflicts should be solved among the parties involved in a conflict by negotiations. If the parties involved cannot find a consentaneous solution a third party should be asked for mediation (cf. Dahrendorf 1961; Glasl 1992).

Assuming that users of groupware do not always work at the same time or at the same place, technical mechanisms should be offered to support them in solving conflicts caused by the activation of a function. They could make conflicts visible to users affected and offer a channel for communication among them. To offer a base for communication the user affected should have the right to intervene against the activation of a function. In case of disagreement among users these mechanisms could contain a strategy for mediation.

Though technical support seems to be crucial to regulate conflicts in groupware, social practice in handling conflict should not be replaced (cf. Dourish 1993). Therefore, we will present technical mechanisms to handle conflicts which can be embedded in social practice in different ways. Thus, they allow even for modes of conflict regulation which are not in accordance with the mainstream of conflict theory. 'The technical mechanisms for conflict management can be classified whether they allow the user affected to intervene against an activation, whether they make a conflict visible and whether they offer a channel for communication. Figure 1 gives a classification scheme for these mechanisms.

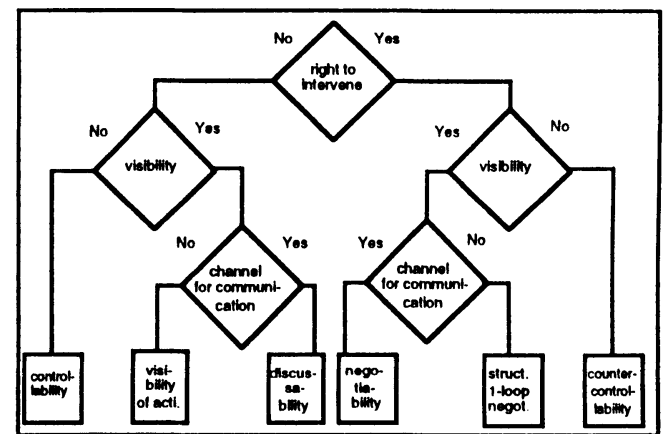

Figure 1: Classification scheme for technical mechanisms to moderate conflicts between activator and affected users

In case of controllability, there is not any technical mechanisms to support users in regulating a conflict. It is either intended to allow the activator to decide on usage of the function autonomously or conflict regulation fully depends on social practice.

In case of visibility of activation the user affected automatically get a notification about the activation of a

\footnotetext{
'This is of special importance as recent research in the field of $\mathrm{CSCW}$ has questioned the validity of the assumptions in mainstream conflict theory (cf. Egger and Wagner 1992).
} 
function which causes conflicts. This information can be either sent to him or be kept available for his access. During the design or configuration of this mechanism the exact content of the data set has to be determined. In case it is not sent to the user affected, the duration of its accessibility has to be decided, too. Thus, this mechanism makes endemic conflict overt which is a precondition for its solution.

Visibility of activation is realized for instance in the RAVE video communication systems. Whenever a channel is built up to another person's office, a special sound is automatically produced to inform the user affected (cf. Gaver 1991, p. 305). Moreover, visibility of activation can be reached by a pop-up message on the workstations screen (cf. Dourish 1993, p. 129). If the user affected is in his office at the moment the channel is built up he can adapt his behaviour accordingly. Though he cannot intervene against the channel by technical means, he may be able to move out of the focus of the camera or redirect the focus of the camera. Thus, the right to intervene might be achieved through social practice. Nevertheless, in certain situations it might be annoying for the users affected to adapt their own behaviour to someone's activation of a function.

Another example for visibility of activation as means for conflict regulation is given by Condon (1993, pp. 180). Conflicts which are coming up when two users attempt to change the same object at the same time on a shared whiteboard are handled by displaying each person's action on the other's screen. As the application provides an additional video channel for the users working on the whiteboard, they can use this channel of communication to negotiate a solution of the conflict. As there exists a means for communication within the application in this case visibility of activation may initiate a negotiation process. If there is not a consentaneous solution different versions of the same object can be stored.

Beyond making a conflict overt, technical support for its communicative solution could be provided. If the activation of a function has become visible by technical means, a technical channel of mutual communication could be built up in that moment. Such a channel can be realized in different ways. The messages transmitted via this channel of communication among the users involved could be structured, semi-structured or unstructured (cf. Malone et al. 1988). Structured communication would just allow to express the intended alternative for use or tailoring of a function. Thus, in this case just highly structured acts of communication would be offered to the users. Additional to these highly structured acts a semistructured channel would allow to the users involved to express further explanations on the desired alternatives. In this sense the co-ordinator (cf. Winograd 1988) offers a semi-structured channel of communication. Contrary to these types of communication channels, an unstructured version would allow to the users involved to provide their point of view without following strict formal patterns. According to the application and its environment such an unstructured channel could be based on textual, audio or video communication. It could be synchronous or asynchronous. For instance in case of a conflict a telephone or a video communication line could be built up among the users involved. Thus, a channel of communication makes a conflict always visible to users affected by technical means.

As long as technical mechanisms are restricted to give visibility to the users affected and to build up a channel for mutual communication, we will talk about discussability. Discussability could be implemented in the following way. The functional alternative is executed immediately and the users affected are technically supported to establish a communication channel with the activator. By this means they may convince the activator to modify the state-transition performed. As the way a conflict is solved strongly depends on how well the users affected can express his argument, a semi-structured or unstructured version of the communication channel should be applied. Right now discussability has not yet been implemented for conflict regulation.

If the users affected have additionally the chance to intervene against the activator's decision by technical means we will talk about negotiability (cf. Herrmann 1995; Wulf 1995). In this case the activation of a function will only take place if activator and users affected have found an agreement. In case of structured and semi-structured negotiability the speech acts are clearly defined by the structured part of each message. Therefore, it is technically simple to detect the outcome of a negotiation and to realize the right to intervene of the user affected. In case of unstructured negotiability there is not any data available for the technical mechanism to detect the outcome of a conversation between activators and the users affected. To realize their right to intervene against the activators decision, in this case the negotiation mechanism should be designed in a way that the users affected will have to input the result of the conversation.

In case activator and user affected cannot find a consentaneous solution within a certain period of time, 
strategies for mediation have to be implemented. Assuming that during the application of groupware a third party for mediation will not always be available, the negotiation mechanism has to be configured in advance in a way that it will activate a default option according to the state of the conversation (cf. Wulf 1995).

Negotiability have not yet been widely implemented. Hammer et al. (1993, p. 143) propose to handle conflicts concerning the activation of the forwarding function in in-house telephone systems by unstructured negotiability. Whenever the activator wants to establish a call forwarding, a telephone line will be built up automatically to the user affected. Via this line they can negotiate, finally the user affected has to confirm the activation. In this case the negotiation process is entirely supported by technical means. The users involved are forced to communicate with each other to find a solution of a potential conflict. The default option in case of disagreement is selected by the user affected - the forwarding function will not get active.

A special case of negotiability is the structured single loop version. In this case communication among users is not technically supported. The user affected just gets a notification on the activator's decision. He can intervene against this decision by technical means expressing a disagreement. Again strategies for mediation have to be realized to handle cases where a consentaneous solution cannot be found within in a certain period of time.

Structured single loop negotiability as a means for conflict management has been proposed for instance in video communication systems. Dourish (1993, pp. 128) presents it as a solution to privacy problems. A channel is only built up when the user affected has agreed on it by pushing a button. Similar solutions were described by Cool et al. (1992) and Belotti and Sellen (1993). This mechanism is already well known in ordinary telephone systems. A telephone line is just established when the recipient (user affected) has agreed on it by picking up the receiver.

The final type of technical mechanisms for conflict regulation is called counter-controllability. In this case the activation of a function is neither made visible to the uscrs affected by technical means nor are the users affected supported to negotiate with the activator via a technical channel of communication. Nevertheless users affected get technical support to intervene against the activation of a certain function. They are equipped with another function whose state transitions will be checked whenever the activator tries to activate function F. If the state transitions resulting from the activation of the counter-controlling function allow for the intended activation, it will be executed immediately, else the function which was supposed to be activated will take a default state in case of disagreement among the users. Thus, contrary to all the other mechanisms proposed so far the users affected decide about their position held in a conflict before the conflictious function is activated.

Counter-controllability as a means to handle conflicts can be found in several CSCW applications, as well. Dourish (1993) has implemented a program within the RAVE video communication which allows users to cut off incoming video calls automatically. The cut off mechanisms can be specified for certain groups of users and periods of time. Malone et al. (1988) and Terveen and Murray (1996) have proposed a similar mechanism for e-mail systems to filter incoming mails. In these cases counter-controllability rather contributes to an automatic solution of a conflict than encouraging negotiation. As it hinders to establish a channel of communication, a negotiation had to be performed by the help of another communication channel. This strict option of conflict solution was intended by the designer of these mechanisms to protect the users affected right for privacy and communicative self-determination.

\section{REQUIREMENTS FROM THE POLITEAM PROJECT}

In the following we want to show how these concepts were applied within a groupware development project. Within the POLITeam Project we develop a groupware application in an evolutionary and participative way. Its functionality mainly consists of an electronic circulation folder, shared workspaces, and an event notification service. Based on the experiences gained by introducing a commercial product (LinkWorks by DEC) in three different fields of application, we develop advanced versions of the system (Klöckner et al. 1995, Sohlenkamp et al. 1997). An important means for user involvement are different types of workshops in which users, support staff, and designers participate. In the design-centered workshops users and support staff report on their experiences and articulate requirements, to which the designers respond by presenting their concepts for the next version of the system. Conflicts among users caused by the activation of a function have been discussed within several of these workshops.

Conflicts concerning the activation of the deletefunction were discussed within a section of a federal ministry. This workgroup had decided to build up a 
folder to share mailing lists for different groups of recipients. Usually, one of the members of the unit started to build up such a list and made it available for his colleagues by storing it in the shared folder. The colleagues used and updated the list for their purposes. By this work practice, the folder became more and more crowded. Thus, the users felt a need to delete mailing lists which were not used any more. Nevertheless, whoever would do so, would violate the interests of those users who were still using the list.

After the users had brought up that topic within the workshop, a designer proposed verbally certain of the mechanisms developed in section 2 to be integrated in the next version of the system. It was generally agreed among the users that mechanisms which provided a technical channel for communication (negotiability and discussability) were not needed. As the offices of the users of this section are located around the same hallway, technical channels of communication would replace face-to-face contact which was regarded as undesirable. Counter-controllability would force each individual user to specify in advance whether a certain mailing list should get protected from being deleted. There were concerns whether these a priori specifications would represent the users' intention in the moment a certain list should get deleted.

A structured single loop version of negotiability which gave any group member the right to veto the execution of the delete function was discussed controversially among the uses involved. One user assumed that such a mechanism would lead to a further bureaucratisation of his work, if anybody had to agree before. He therefore preferred just receiving before hand a notification (visibility of activation). One other user feared the additional workload resulting from permanent decision making. Other users liked the idea that the application of this mechanism would allow all the users affected to participate in the decision making ("It is helpful to get an okay. In this case you know what is the matter"). As a result of this discussion, the designers proposed a variant of structured single-loop negotiability. The mechanism for conflict regulation should give the users affected a longer period of time (e.g. one week) to react to the activator's decision. If individual users do not react by then, their agreement would be taken as default option in case of time delay. The voting rule should give any member of the group a veto-right to intervene against the execution of the delete function. Such a configuration was generally accepted by the members of the group. As technical mechanisms for conflict-regulation were not available in that system version, the members of the group agreed upon sending a mail before activating the delete function on one of the mailing lists. Thus, a social convention was used to replace technical mechanisms.

Within another workshop we presented a new function to the members of the same group. The new function allows to the creator of an object to stop objectsharing with other users. This function had been required to handle documents which had to be shared just for a short period of time within a group. During the workshop the users mentioned other types of documents on which they would not like the creator to determine the level of object-sharing. For instance, the head of the unit argued that most of the documents were created by one of his colleagues while he would just modify or read them before sending them out. His interests were affected by this function because he could not reaccess these documents after its activation by the creator. Concerning certain type of documents he demanded to equip this function with structured single-loop negotiability. For other documents visibility of activation seemed sufficient to him to protect his interests.

Presenting a newly designed search tool in a workshop, we found conflicts between the activator and the users affected, too. The participants of this workshop belonged to a different field of application - an organizational unit of a state ministry. Th $s$ search tool presented allows the activator to specify certain criteria to search for document in the whole groupware system. The result of the search is displayed in a window. Out of this window, the activator can manually select relevant documents. These documents will be displayed on his desktop even if they were not accessible for the activator before. Thus, this function allows the activator to access other peoples documents.

Obviously such a design affects the interests of the users affected. For instance one user imagined that his boss would sit at night on his computer searching for documents containing certain keywords. Therefore, all of the users asked for counter-controllability to be able to create private domains where other users' access via the search-tool would be impossible. This mechanism was already realized in the prototype presented. Moreover, they asked for visibility of activation in case of nonprivate documents got shared as a consequence of the activation of the search-tool.

Stating that for certain types of dodtuments (e.g. circulation folders) search activities should not result in document-sharing, they criticised the a lack of technical flexibility of the search-tool. Concerning this type of 
documents they rather preferred to provide for the activator only a copy or just some information on the document's state (e.g. name of the current owner). Only in case the user affected had explicitly agreed on document sharing, this functional alternative should be activated. Thus, they proposed a structured single loop version of negotiability where the intermediate state and the default option provided the activator with restricted possibilities while documents sharing was just executed after an agreement of the user affected. The user asked to he able to choose the intermediate state and the default options in a way that they should still provide enough information to allow the activator to continue his tasks without waiting for the users affected reaction.

\section{APPROACH FOR IMPLEMENTATION}

The experiences from the POLITeam project show that there are different functions of the application which cause conflicts among users. For sake of conformity with user expectations, mechanisms which support users to regulate conflicts concerning the activation of these functions should be designed in a similar way. Furthermore, an implementation architecture for groupware should offer technical flexibility. Thus, it should ease to tailor the ordinary functionality as well as the linkages between conflict constellations and technical mechanisms for conflict regulation. To support technical flexibility as well as conformity with users' expectations, we implemented mechanisms for conflict regulation centrally in a component apart from the functions they refer to (conflict manager). Thus, instead of implementing these mechanisms for every function separately, all the function of an application, which contains a potential for conflict, are just equipped with configuration data which indicate the mechanism to apply. These data have to be created and stored for each conflict constellation in the configuration store.

The structure of the configuration data has to be determined for each function and allows to specify the mechanism applied depending on the actual context of a function's usage. In case of the delete function in POLITeam the context of usage consists just out of the name of the document to be deleted. In this architecture the linkage between a conflict constellation of a function and a technical mechanism for conflict regulation is tailored by just modifying the configuration data. Technical mechanisms themselves could be tailored centrally for the whole application.

Based on these considerations, we have implemented the technical mechanisms for conflict regulation in the way displayed in figure 2 . LinkWorks offered an application programming interface which we used to extend the code of the three functions in a way that after the activation and hefore the execution of the conflictious state transition the technical mechanisms for conflict regulation are executed (extension of the function).

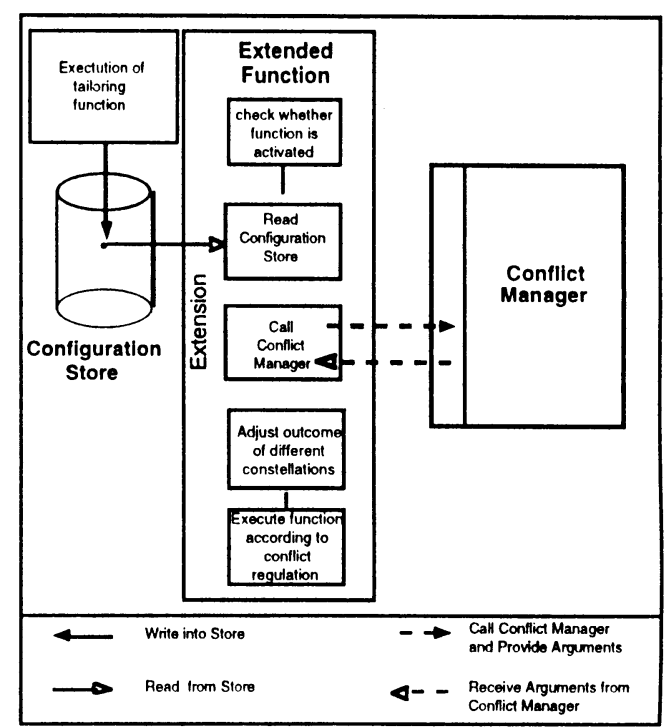

Figure 2: Overview of an architecture to implement technical mechanisms for conflict regulation

Thus, whenever a user activates one of the three functions the relevant configuration data for each of the function's conflict constellations is read from the configuration store. The conflict manager is called and the functional alternative resulting from the conflict regulation is sent back to the process initiated by the extended function. If a function contains more than one conflict constellation, the solutions worked out within the different constellations have to be adjusted according to predefined rules. Finally, the resulting functional alternative determined within this process of technically supported conflict regulation will be executed.

In section 2 , we assumed that the role of the user affected was just held by one individual. This assumption is not always given. For instance, when activating the delete function in POLITeam depending on the document more than one user may be affected in the same way. In this case, we just have to handle a single conflict constellation, where a group of users is in 
the role of the user affected. Such groups have to he taken into consideration when designing technical mechanisms to regulate conflicts (cf. Wulf 1995). To satisfy the user requirements concerning the "delete"function, a group-oriented version of the mechanism: single loop structured negotiability was implemented in the conflict manager which transformed the individual users' reaction into a group's decision. In case of the search tool a group-oriented version of the mechanism visibility of activation grants notifications to a whole group of users affected.

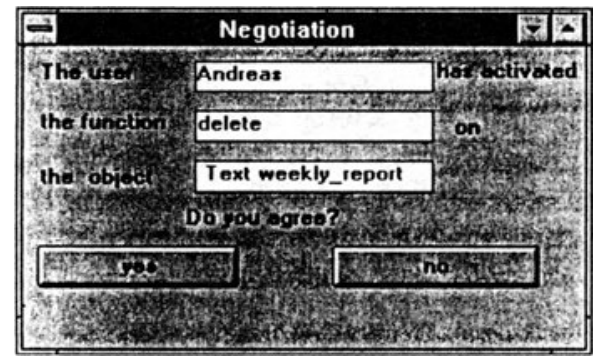

Figure 3: Negotiation window received by the users affected of the "delete"- function

Taking the example of the "delete" - function, we will describe how the mechanisms for conflict regulation are seen from a user's point of view. If a user tries to delete an address-list, he is notified by a system created message indicating that the document can only be deleted in case none of the other users disagrees within a weeks span. Then the conflict manager sends a negotiation window to the users affected (cf. figure 3 ). They receive it in a especial mail folder for conflict handling. If they open this folder, they see all the messages generated by the conflict manager. They can access and react to them individually. If all of the users affected have reacted the activator receives a message which tells him depending on the result either that the deletion is not possible or to click a button to finally delete the document.

\section{CONCLUSION}

We have proposed a classification scheme for technical mechanisms which support users in regulating conflicts concerning the activation of certain groupware functions. Discussions with users in workshops showed that the technical mechanisms presented in this paper are a useful means in regulating conflicts between users. Therefore, we have implemented a first prototype.
As a next step, the design of these mechanisms has to he evaluated hy the users. The prototypical implementation of additional mechanisms effects the performance of the application. Therefore, further research towards efficient implementations is needed. Furthermore, we have to support the configuration activitics by an appropriate user interface. Assuming that there are even more potentially conflictious functions within an application and for each of their conflict constellations a configuration matrix has to be filled, there is an obvious need for innovative approaches which allow to reuse configurations for conflict regulation.

Looking at the implementation architecture for technical mechanisms of conflict regulation. it resembles that one of access control. Indeed, approaches to implement access rights in groupware distinguish more than just reading and writing permissions. They allow to specify access rights concerning a wide variety of functions (cf. Shen and Dewan 1992). To realize access control, one has to extend the code of each function in a similar manner as we have proposed. Access control lists used in these approaches can be seen as configuration data for conflict regulation. Nevertheless, compared to the approach proposed in this paper, access control in groupware has just regarded counter-controllability as a technical mechanism for conflict regulation. Thus, our results would plea to extend these approaches to offer users more than just a single technical mechanism for conflict regulation.

\section{ACKNOWLEDGEMENTS}

I would like to thank Andreas Pfeifer and Markus Rohde for valuable discussions on the topics addressed in this paper. Dirk Fischer has implemented the prototype. This research has been supported by the German Ministry of Education and Research within its "Polikom" research program under the grant No. 0I QA $405 / 0$.

\section{REFERENCES}

Belotti, V.; Sellen, A.: Design for Privacy in Ubiquitous Computing Environment, in: de Michelis, G.; Simone, C.; Schmidt. K. (eds): Proceedings of the Fourth Conference on Computer Supported Cooperative Work - ECSCW '93. Kluwer, Dordrecht, 1995, pp. 77 - 92

Cool, C.; Fish, R.S.; Kraut, R.E.; Lowery, C.M.: Interactive Design of Video Communication Systems, in: CSCW '92. Sharing Perspectives. Proceedings of 
the Conference on Computer-Supported Cooperative Work, (ACM Press, New York) 1992, pp. 25 -32

Dahrendorf, R.: Elemente einer Theorie sozialen Konflikts, in: Dahrendorf, R. (ed.): Gesellschaft und Freiheit, München 1961, pp. 197 - 236

Dourish, P.: Culture and Control in a Media Space, in: de Michelis, G.; Simone. C.; Schmidt, K. (eds): Proceedings of the Third Conference on Computer Supported Cooperative Work - ECSCW '93, Dordrecht, NL, Kluwer 1993, pp. 125 - 138

Condon, C.: The Computer Won't let me: Cooperation, Conflict and the Ownership of Information, in: Easterbrook, S. (ed.) CSCW: Cooperation or Conflict, Springer, London u. a. 1993, pp. $171-185$

Easterbrook, S. M.; Beck E. E.; Goodet, J. S.; Plowman, L.; Shaples, M.; Wood, C. C.: A Survey of Empirical Studies of Conflict, in: Easterbrook, S. (ed.) CSCW: Cooperation or Conflict, Springer, London et al. 1993, pp. 1 - 68

Egger, E.; Wagner, I.: Time Management: A Case for CSCW, in: CSCW '92. Sharing Perspectives. Proceedings of the Conference on Computer-Supported Cooperative Work, ACM-Press, New York 1992, pp. $249-256$

Gaver, W.: Sound Support for Collaboration; in: L. Bannon, M. Robinson; K. Schmidt, (eds.): Proceedings of the Second European Conference on Computer Supported Cooperative Work, Amsterdam, 1991, pp. $276-292$.

Glasl, F.: Konfliktmanagement, 3rd ed., Bern 1992

Greenberg, S.: Personizable Groupware: Accomodating individual roles and group differences, in: L. Bannon; M. Robinson; K. Schmidt (eds.): Proceedings of the Second European Conference on Computer Supported Cooperative Work, Amsterdam, 1991, pp. 17 - 31.

Hammer, V.; Pordesch, U.; Roßnagel, A.: Rechtliche Gestaltungsanforderungen an betriebliche ISDNTelefonsysteme, Berlin Heidelberg, Springer, 1993

Herrmann, T.: Workflow Management Systems: Ensuring Organizational Flexibility by Possibilities of Adaptation and Negotiation, in: Proceedings of COOCS, August 13 - 16, 1995, Milpitas, CA, ACMPress, New York 1995, pp. 83 - 96.

Kahler, H.: Von der Empirie zur Gestaltungsanforderung - Beispiele für den Einfluß explorativer Empirie auf die Entwicklung von Gestaltungsanforderungen für Groupware, in: Hartmann, A.; Herrmann, Th.; Rohde, M.; Wulf, V. (eds): Menschengerechte Groupware - Software-ergonomische Gestaltung und partizipative Umsetzung, Stuttgart, Teubner 1994, pp. 109-124

Klöckner, K.; Mambrey, P.; Sohlenkamp, M.; Prinz, W.; Fuchs, L. Kolvenbach, S.; Pankoke-Babatz, U.; Syri, A.: POLITeam - Bridging the Gap between Bonn and Berlin for and with the Users, in: H. Marmolin; Y. Sundblad; K. Schmidt (eds.): Proceedings of the Second European Conference on Computer Supported Cooperative Work, kluver, Dordrecht, 1995, pp. 17 32

Malone, T. W.; Grant K. R.; Lai, K.-Y.; Rao, R.; Rosenblitt, D.: Semistructured Messages are Surprisingly Useful for Computer-Supported Coordination, in: I. Greif (ed.): CSCW: A Book of Readings, Morgan-Kaufmann Publisher, San Mateo, California, 1988, pp. 311 - 334

Schmidt, K. : Riding a Tiger or Computer Supported Cooperative Work, in: L. Bannon, M. Robinson; K. Schmidt, (eds.): Proceedings of the Second European Conference on Computer Supported Cooperative Work, Amsterdam, 1991, pp. 1 - 16

Shen, HH. and Dewan, P.: Access Control for Collaborative Environments, in: Computer-Supported Cooperated Work '92. Sharing Perspectives. Proceedings of the Conference on ComputerSupported Cooperative Work, ACM Press, New York, pp. $51-58$

Sohlenkamp, M.; Fuchs, L.; Klöckner, K.; Kolvenbach, S.; Mambrey, P.; Pankoke-Babatz, U.; .; Prinz, W.; Syri, A.: Supporting the Distributed German Government with POLITeam, in: International Journal on Multimedia Tools and Applications - Special Issue on Multimedia Projects in Governments, 1997 (to appear)

Terveen, L.; Murray, L.: Helping Users Program Their Personal Agents. In: Proceedings of CHI '96, April 13.-18, ACM-Press, New York 1996, pp. 355-361

Winograd, T.: A Language/Action Perspective on the Design of Cooperative Work, in: I. Greif (ed.): CSCW: A Book of Readings, Morgan-Kaufmann Publisher, San Mateo, California, 1988, pp. $311-334$.

Wulf, V.: Negotiability: A Metafunction to Tailor Access to Data in Groupware; in: Behaviour \& Information Technology, Vol. 14, No. 3, 1995, pp. 143 $-151$

Wulf, V.: On Conflicts and Negotiation in Multi-User Applications; in: Kent, A.; Williams, J. G. (eds): Encyclopedia of Microcomputers, Dekker, New York et al., 1997 (invited paper) 